\title{
Upregulation of emotion areas through neurofeedback with a focus on positive mood
}

\author{
Stephen Johnston • D. E. J. Linden • D. Healy • \\ R. Goebel • I. Habes • S. G. Boehm
}

Published online: 25 November 2010

(C) Psychonomic Society, Inc. 2010

\begin{abstract}
Real-time functional magnetic resonance imaging can be used to feed back signal changes from the brain to participants such that they can train to modulate activation levels in specific brain areas. Here we present the first study combining up-regulation of brain areas for positive emotions with psychometric measures to assess the effect of successful self-regulation on subsequent mood. We localized brain areas associated with positive emotions through presentation of standardized pictures with positive valence. Participants up-regulated activation levels in their target area during specific periods, alternating with rest. Participants attained reliable self-control of the target area by the last of three seven-minute runs. This training effect was supported by an extensive network outside the targeted brain region, including higher sensory areas, paralimbic and orbitofrontal cortex. Self-control of emotion areas was not accompanied by clear changes in self-reported emotions;
\end{abstract}

Stephen Johnston and D. E. J. Linden contributed equally to the project.

S. Johnston $(\square)$

School of Social Sciences, Psychology Department,

Brunel University,

Uxbridge, Middlesex UB8 3PH, UK

e-mail: Stephen.Johnston@brunel.ac.uk

D. E. J. Linden $\cdot$ S. G. Boehm

Wolfson Centre for Clinical and Cognitive Neuroscience,

School of Psychology, Bangor University,

Bangor LL57 2AS, UK

D. Healy

North Wales Department of Psychological Medicine,

Cardiff University,

Bangor LL57 2PW, UK

R. Goebel $\cdot$ I. Habes

Department of Cognitive Neuroscience, Maastricht University,

MD-6200, Maastricht, The Netherlands trend-level improvements on depression scores were counteracted by increases on measures of fatigue, resulting in no overall mood improvement. It is possible that benefits of self-control of emotion networks may only appear in people who display abnormal emotional homeostasis. The use of only a single, short, training session, overlap between positive and negative emotion networks and aversive reactions to the scanning environment may have prevented the detection of subtle changes in mood.

Keywords Real-time fMRI · Neurofeedback · Emotion · Mood

\section{Introduction}

FMRI (functional magnetic resonance imaging)-based neurofeedback (deCharms et al., 2005; deCharms, 2007; Weiskopf et al., 2004a, b) is a tool to train participants to regulate their own brain activity with high spatial precision. It has been applied to motor cortex (deCharms et al., 2004), sensory areas (Yoo et al., 2006), insula (Caria et al., 2007), inferior frontal cortex (Rota et al., 2009), the anterior cingulate (deCharms et al., 2005; Weiskopf et al., 2003) and the amygdala (Posse et al., 2003). One of the most exciting uses of this approach was demonstrated by deCharms et al. (2005) who used neurofeedback to alter participants' perceived levels of pain in response to a noxious thermal stimulus. deCharms trained participants to control the activity levels in rostral anterior cingulate (rACC), a region known to be involved in the perception and regulation of pain. Successful up-regulation of rACC while the noxious thermal stimulus was administered resulted in subsequently higher reports of pain; conversely, down-regulation of rACC during painful stimulation resulted in lower ratings of pain. 
Recently, we applied this approach to examine the efficacy of this technique for training self-regulation of emotional networks. We trained participants to upregulate target areas in inferior frontal and insular cortex and the amygdala, whereby each region was localized through their response to negative affective pictures (Johnston, Boehm, Healy, Goebel, \& Linden, 2010). In order to probe the potential use of this technique for mood improvement in clinical settings, we now aimed to achieve the same with areas responsive to positive affect. Although any type of self-control of emotion networks, even that achieved by enhancing areas responsive to negative affect, may increase patients' experience of self-efficacy and thus be a helpful adjunct to the cognitive therapy of depression, a pathway through positive emotion networks seemed both clinically and ethically preferable. We therefore applied the same localizer procedure as in Johnston et al. (2010), using positive, negative and neutral standardized stimuli from the International Affective Pictures System (Lang, Bradley, \& Cuthbert, 1999), but now used the area that responded most significantly to positive pictures as the target area. Participants then trained to upregulate this area in three consecutive runs of ca. seven minutes each during a single scanning session. In order to assess any changes in mood that might be induced by successful neurofeedback, we obtained standard self-report measures of current mood state before and after the session. To confirm that any training effects were due to the use of neurofeedback-guided learning, as opposed to practice effects, a second group of participants took part in a control condition that involved the same procedure, but this group received no feedback information.

\section{Methods}

\section{Participants}

Thirty-one volunteers were recruited from Bangor University staff and postgraduate students and paid $£ 20$ each for participation. Twenty-one formed our experimental group and received neurofeedback information during their runs, but because of technical problems (gradient instability leading to irremediable artifacts) only data for 17 participants could be entered into the final analysis (nine females, eight males, age range 21-54; all right-handed). The remaining ten participants constituted the control group and received no feedback during their sessions (five females, five males, age range 20-41; all right-handed). All participants had normal or corrected to normal vision and no history of neurological or psychiatric illness. They participated in the experiment after giving informed consent. The experimental protocol was approved by the ethics committees of the School of Psychology, Bangor
University, and the North West Wales NHS Trust. All participants were debriefed after the experiment about their strategies.

\section{Procedures}

The basic method was identical to that used in Johnston et al., 2010. We administered the Profile of Mood States (POMS, Lorr, McNair, \& Droppleman, 1971) and the Positive and Negative Affect Scale (PANAS, Watson, Clark, \& Tellegen, 1988) immediately before and after the neurofeedback session.

We acquired fMRI data on a 3 Tesla Philips Achieva system $(\mathrm{TR}=2 \mathrm{~s}, \mathrm{TE}=30 \mathrm{~ms}, 30$ slices, 3 -mm slice thickness, inplane resolution $2 \times 2 \mathrm{~mm}$ ). In order to localize emotionresponsive brain areas, we used pictures from the International Affective Pictures System (IAPS) (Lang et al., 1999) with positive (mean normative ratings for valence 6.90 [SD .55], arousal 6.00 [SD .74]), negative (valence 2.8 [SD .42], arousal 5.63 [SD .55]) and neutral valence (valence 5.45 [SD .56 ], arousal 3.44 [SD .47]). We presented 12, 6-s blocks (four pictures, each presented for $1.5 \mathrm{~s}$ ) per category in a pseudorandom order, alternating with a fixation baseline. We computed an online general linear model (GLM) after motion correction with three predictors corresponding to the three picture categories, convolved with a hemodynamic reference function, using the Turbo-BrainVoyager software package (Brain Innovation B.V., Maastricht, the Netherlands). We used the brain area with the highest effect for positive vs. neutral pictures as the target area. We chose this approach, rather than the contrast between positive and negative images, because of the considerable overlap between emotion-responsive areas (Fig. 1) and because sensitivity, rather than specificity, was the main aim for identifying potential neurofeedback-responsive areas. Target areas for the experimental group were in the right $(n=4)$ and left $(n=1)$ ventrolateral prefrontal cortex (VLPFC) right $(n=$ $1)$ and left $(n=1)$ dorsolateral PFC (DLPFC), right $(n=4)$, left $(n=3)$ and bilateral $(n=1)$ insula, and the right $(n=1)$ and bilateral $(n=1)$ medial temporal lobe. Target areas for the control group were in the right $(n=3)$ and left $(n=3)$ ventrolateral prefrontal cortex (VLPFC), right IFG $(n=2)$, right $(n=1)$ and bilateral $(n=1)$ insula. Each ROI was constructed such that the entire extent of the anatomical region that exhibited contiguous super-threshold levels of activation was included in the axial plane, and across three functional imaging slices. Since the main criteria for success in the neurofeedback task was the successful regulation of the ROI, no strict definition of ROI size and shape was imposed.

The 11-min localizer run was followed by three 7-min neurofeedback runs. Participants were instructed to upregulate their target region activity for periods of $20 \mathrm{~s}$ ("up"), alternating with baseline periods of $14 \mathrm{~s}$ ("rest") (12 up-rest 
Fig. 1 Four coronal slices show the areas responsive to negative (red), positive (green) and neutral (blue) emotions. Overlap between emotion maps is denoted through color additions according to the Red-GreenBlue (RGB) system (for example yellow: overlap between positive (green) and negative (red) emotions)

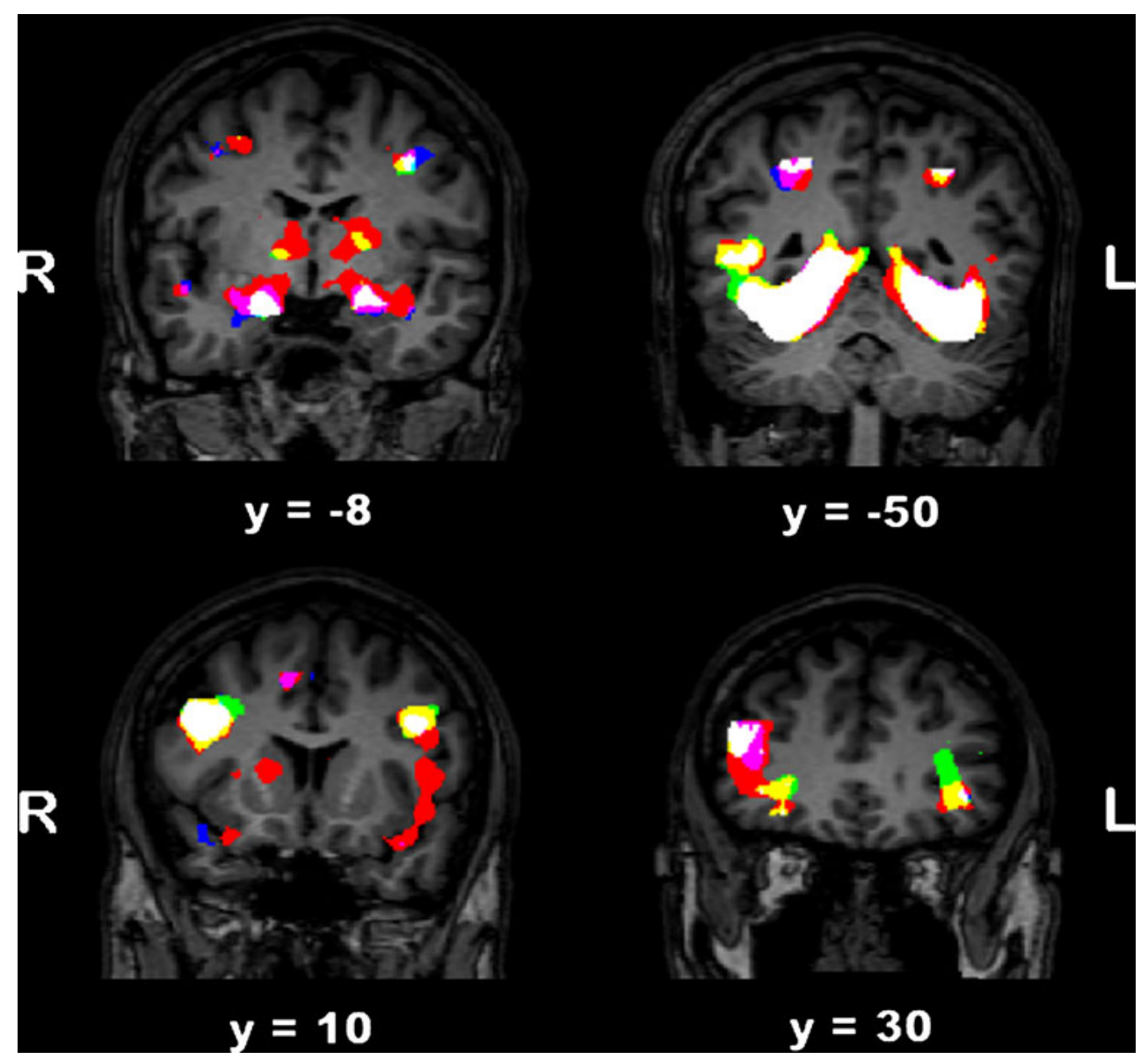

cycles per run). Participants were informed about the way in which the target area had been identified but not prescribed a strategy. For the experimental group, who received information on the activity level of their ROI, continuous feedback (updated every TR) was displayed using the picture of a thermometer whose temperature reflected amplitude increases of the fMRI signal in the target area. For the control group, the same instruction was given, the exception being that the thermometer display was kept constant at a 50\% level at all times during the run (i.e., no feedback). Control participants were aware that no feedback would be provided.

\section{Data analysis}

The identification of target areas and the feedback of signal changes rely on the online computation of incremental GLMs (after motion correction). For the localizer runs, the online GLM was computed using three predictors that modeled the valence type of the presented images (i.e., positive, negative and neutral), convolved with a hemodynamic reference function. For the neurofeedback runs, we computed the online GLM with one predictor for the regulation state (up, rest), convolved with a hemodynamic reference function. For further group analysis, we per- formed the customary steps of three-dimensional fMRI analysis using the BrainVoyager QX software package, including high pass filtering using a GLM approach with a Fourier basis set ( 2 sine/cosine pairs), spatial filtering with a $4 \mathrm{~mm}$ full width at half maximum Gaussian kernel and normalization into the Talairach coordinate system (with new cubic voxel dimensions of 2 -mm edge length). The six motion parameters (for translational and rotational movement) were added as confounds to the localizer and neurofeedback models.

To assess the effectiveness of the neurofeedback procedure versus no-neurofeedback, we compared beta values for the target region using mixed analysis of variance (ANOVA), with the between subjects factor being Group (Feedback vs. No-feedback) and a within subjects factor of Run (First (1) vs. Final (3)). For the experimental, 'Feedback' group, we additionally computed group maps for the emotion localizer and overlaid effects for the three categories thresholded at $p<.05$ (False Discovery Rate, FDR corrected at $p<.05$ ) (Fig. 1). We also computed group maps for the neurofeedback runs and analyzed the overall contrast of "up" periods vs. the "rest" baseline (thresholded at $p<.05$, FDR) (Fig. 3, Table 1) and the change of "up" activation from the first to the last run (Fig. 4, Table 2). The latter contrast was thresholded at $p<.05$, corrected at 
Table 1 Effects of neurofeedback

\begin{tabular}{lllll}
\hline Brain area & $\mathrm{X}$ & $\mathrm{Y}$ & $\mathrm{Z}$ & No. of voxels \\
\hline Activation & & & & \\
Left MTL & -18 & -17 & -9 & 6,300 \\
Right MTL & 25 & -14 & -12 & 2,151 \\
PVC & 5 & -84 & 13 & 4,583 \\
Cuneus & -4 & -46 & -2 & 12,969 \\
Left Insula & -27 & 32 & 7 & 1,473 \\
ACC & 3 & -8 & 28 & 2,926 \\
Deactivation & & & & \\
Right TPJ & 48 & -47 & 34 & 4,647 \\
\hline
\end{tabular}

cluster level $\left(400 \mathrm{~mm}^{3}\right)$ based on the Monte-Carlo simulation (Forman et al., 1996) implemented in BrainVoyager QX. For selected regions, we computed event-related average time courses (computing the percent change of the fMRI blood oxygenation dependent [BOLD] signal against a baseline comprising the three time points before each "up" period) for the activation across all neurofeedback runs (Fig. 3) and separately for early and late runs (Fig. 4).

Psychometric data were analyzed with two-way ANOVA with a single factor of group (Feedback vs. No-feedback).

\section{Results}

As in our previous study with the same localizer procedure (Johnston et al., 2010), we found considerable overlap at group level in activation across emotion categories in visual areas, medial temporal lobe, including the amygdala, and prefrontal cortex. Activation in some regions was most extensive for negative images (e.g., in the basal ganglia and insula), but there were sites of highly significant activation for positive images, e.g., in ventrolateral prefrontal cortex (Fig. 1).

The analysis of neurofeedback success in the individually localized target areas showed main effects of Group $F(1,9)=13.9, p<.005$ and Run $F(1,9)=19.7, p<.005$, and a marginally significant interaction of Group $x$ Run $F(1,9)=4.0, p<.075$. Follow-up paired samples $t$ tests revealed that while participants that received feedback about the activity in their ROI achieved higher activation in run 3 compared to run $1(\mathrm{t}(16)=4.089, p=.049$ (twotailed)), indicating improvement with training (Fig. 2a), participants that did not receive feedback, failed to do so $(\mathrm{t}(9)=.671$, n.s., Fig. 2b). Participants reported strategies involving positive memories and imagery to up-regulate their target areas, for example visualizing being with friends and family. The strategies were personalized and often involved episodic memory of past positive events. Participants often reported testing different strategies before settling for the one that worked best.

The whole-brain analysis of activation during the neurofeedback periods for the experimental group revealed activity increases in bilateral MTL, including the amygdala, hippocampus and entorhinal cortex, primary visual cortex (V1) and cuneus, the left insula and the anterior cingulate cortex and a decrease in the right temporoparietal junction (TPJ) (Fig. 3, Table 1).

The comparison of activation levels during the "up" periods between the last and first (and less successful) run showed increases in primary and secondary somatosensory cortex, early and higher visual (V1, middle occipital gyrus (MOG)) and multimodal (STS) areas, prefrontal areas connected with the limbic system (OFC, ACC, insula) and entorhinal cortex (Fig. 4, Table 2). Activation decreased
Table 2 Whole-brain effects of training (last vs. first run)

$P C G$ postcentral gyrus; $E R C$ entorhinal cortex

*This area was included although it did not meet the cluster threshold because of our specific anatomical hypothesis of striatal activation with training success, based on the finding of a very similar cluster in Johnston et al., 2010 (center of mass: $x=9, y=5, z=6$ )

\begin{tabular}{lllll}
\hline Brain area & X & Y & Z & No. of voxels \\
\hline Last > First & & & & \\
Left PCG (vertex) & -7 & -34 & 54 & 650 \\
Left PCG (lateral and ventral) & -34 & -23 & 38 & 3,220 \\
Left OFC & -20 & 40 & 13 & 1,330 \\
Left ACC & -1 & 27 & 22 & 761 \\
Left MOG & -49 & -71 & 1 & 1,116 \\
Left PVC & -5 & -94 & 2 & 896 \\
Left ERC & -27 & -15 & -23 & 611 \\
Right STS & 45 & -34 & 11 & 1,152 \\
Right ERC/ temporopolar cortex & 41 & 6 & -13 & 1,601 \\
Right striatum & 12 & 5 & 11 & $240 *$ \\
First $>$ last & & & & \\
Left VLPFC & -37 & 16 & 25 & 1,126 \\
Right VLPFC & 47 & -5 & 19 & 532 \\
\hline
\end{tabular}




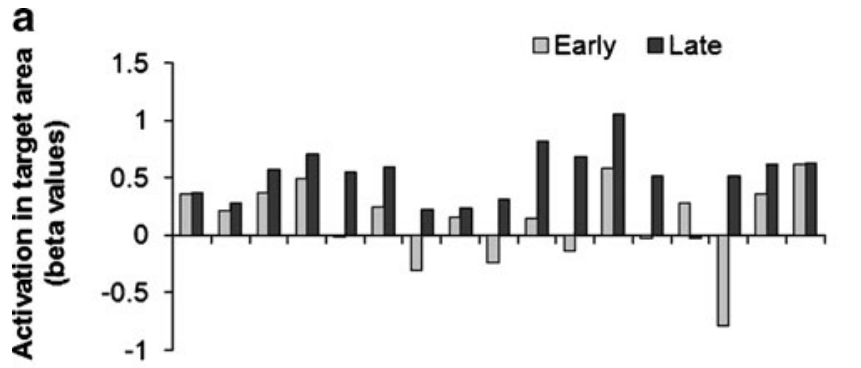

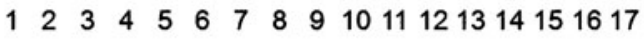

Participant number

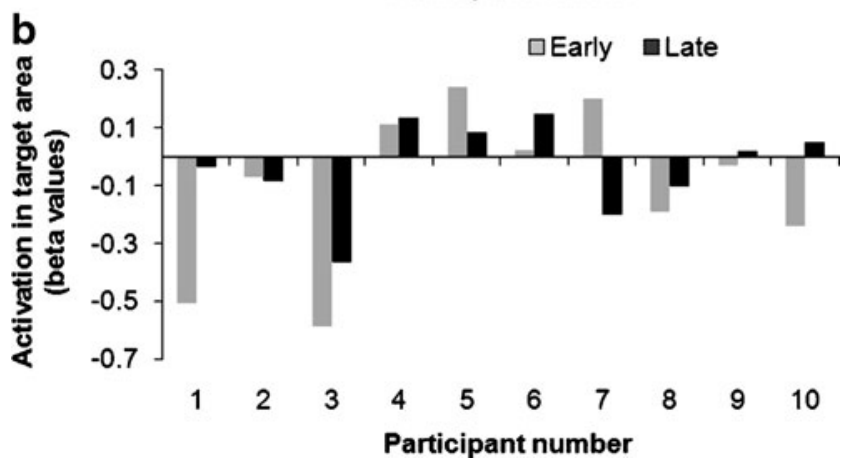

Fig. 2 a Beta estimates for activity in the individual target areas during early and late training for the 17 participants in the experimental group who received feedback. The panel shows that all but one participant had achieved self-control of target area activation by the last training run. b Beta estimates for activity in the individual target areas during early and later training for the ten participants in the control group who did not receive feedback

only in VLPFC areas bilaterally. This may have been an effect of activation in the target area, which often comprised the VLPFC, becoming more focal with training.

The psychometric data did not show any significant mood changes for either the control or the experimental group. Changes on the negative and positive subscale of the PANAS and the POMS total mood disturbance (TMD) score were at $p>.2$. A closer examination of the experimental group, those who received neurofeedback, showed an improvement on the depression subscale of the POMS that came close to significance (decrease from 2.53 to $1.18, \mathrm{t}(16)=1.67, p=.11$ [two-tailed]), but was counteracted by a non-significant increase on the fatigue subscale (4.41 to $5.10, \mathrm{t}(16)=-0.70, p=.50$ [two-tailed]).

\section{Discussion}

Participants in our experimental group, who received neurofeedback, achieved reliable upregulation of the target area, based on positively valenced emotional imagery, in emotion-responsive networks within ca. $20 \mathrm{~min}$. Conversely, participants whose imagery was not guided by the realtime presentation of brain activation, failed to show any improvement in upregulation of their target ROI over the course of the session. This finding supports that of previous authors, e.g., deCharms et al. (2005), who have demonstrated the importance of feedback to inform the strategies used to allow learning of control of the output of discrete cortical regions. The training success of our experimental group confirms our previous results (Johnston et al., 2010), where successful upregulation was attained in cortical areas localized via their response to negative affective pictures. The individually identified target areas in the (mainly ventral) prefrontal cortex/insula and medial temporal lobes were also similar to those areas that were successfully regulated in the previous study. Furthermore the overall network of areas activated across participants and runs during neurofeedback, which included areas in the medial temporal lobe, insula, anterior cingulate cortex (ACC), cuneus and primary visual cortex (Table 1), showed considerable overlap with areas activated during upregulation of areas identified with the negative localizer procedure. This similarity of network-level activation between procedures that involved a positive or a negative localizer (and consequently, strategies that focused on positive or negative emotions, respectively) is suggestive of processes that are not valence specific but rather are involved in evoking multiple types of emotion. Within the limitation of reverse influence (Poldrack, 2006), we might speculate that the whole-brain activation observed reflects memory (MTL), attention (dorsal ACC) and focus on bodily sensations (insula). The deactivation of the TPJ (equivalent to a higher activation during the rest epochs) is compatible with the role of this area in the "default mode network" and its deactivation during attention-demanding processes (Mayer et al., 2010).

One open question is whether the activation of visual cortex is evidence of visual imagery (reported as a key strategy by the participants) or of the subtle changes in the visual display used to convey the feedback. The only way to resolve this issue would probably be to use different sensory modalities for feedback, but this is difficult in the scanning environment because of constraints on auditory and tactile stimulus delivery. However, the observation that sensory areas in other, non-visual modalities (the postcentral gyrus, site of primary and secondary somatosensory cortex, the auditory association field along the superior temporal sulcus) were activated with increasing training success (Table 2) would support an account that is at least partly imagery-based. Other key areas that showed activation increases with training success included parts of a motivation network (rostral ACC, OFC, ventral striatum), which may respond to the rewarding experience of increasing control of the feedback signal, and the entorhinal cortex, a node for the retrieval of long-term memories. Participants reported that their self-control of the target area became more reliable the more they moved from just 
Fig. 3 The left panels show the clusters of highest activation during upregulation periods across participants in the left (upper panel) and right (lower panel) medial temporal lobe. The right panels show the corresponding averaged time courses, with the vertical bars denoting the duration of the "up" period
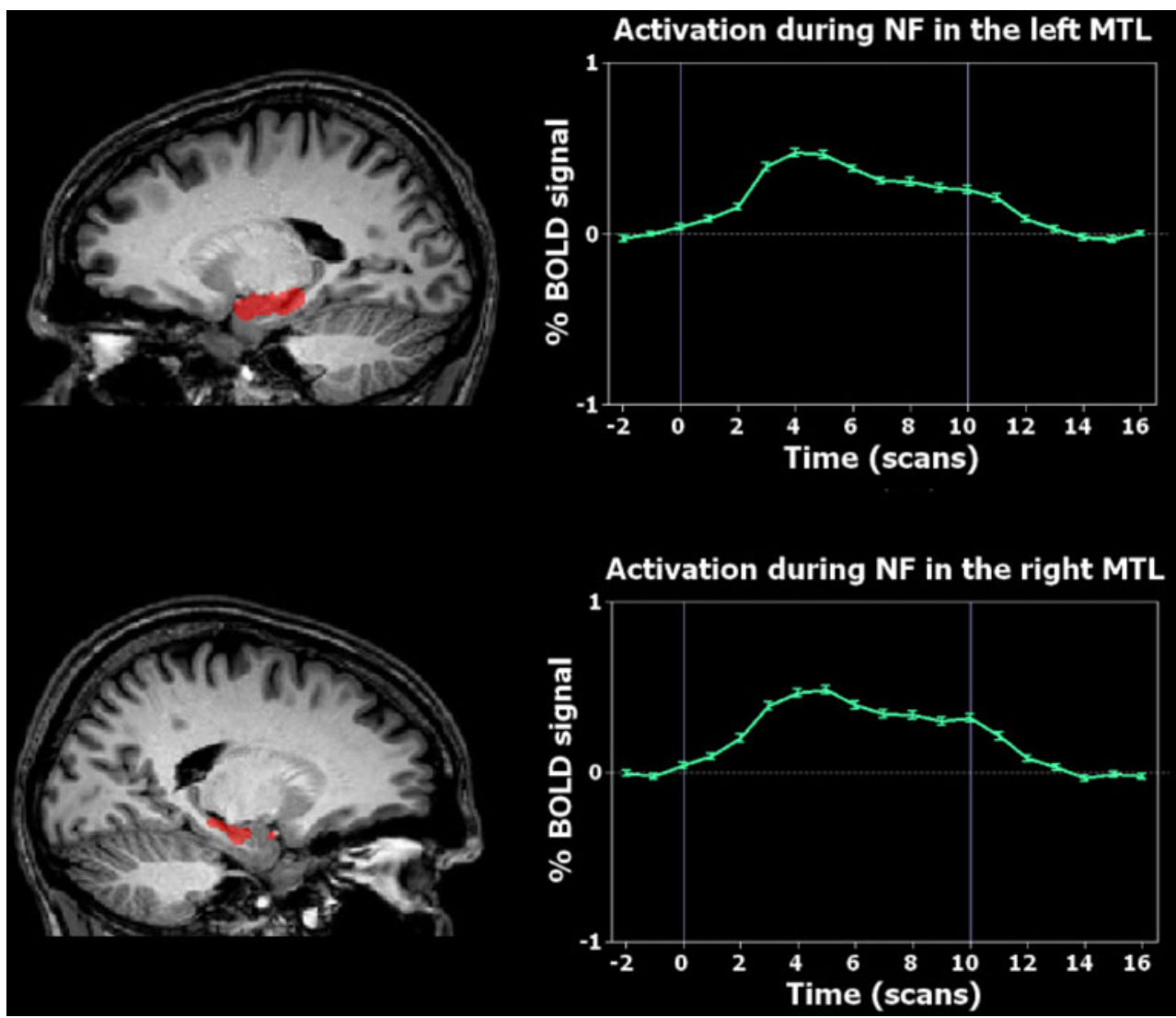

Fig. 4 The contrast map for the upregulation predictor during the last vs. the first runs for each participant shows activation increases (red) in bilateral entorhinal cortex $(E R C)$, right superior temporal sulcus $(S T S)$ and left postcentral gyrus $(P C G)$ and parietal operculum $(P O P)$. The averaged time courses for the right ERC show higher activation for the last (red) compared to the first (green) runs (right lower panel)

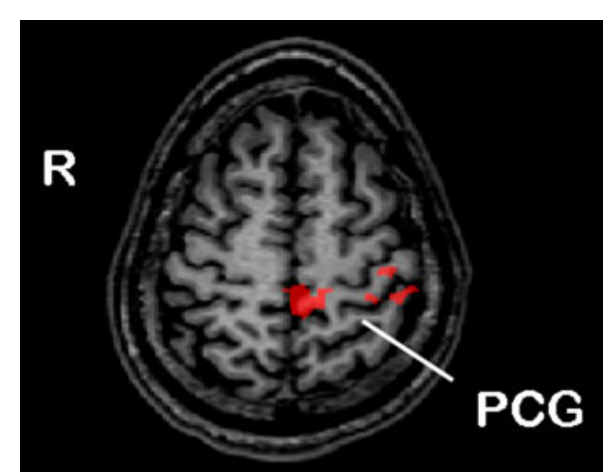

$z=52$

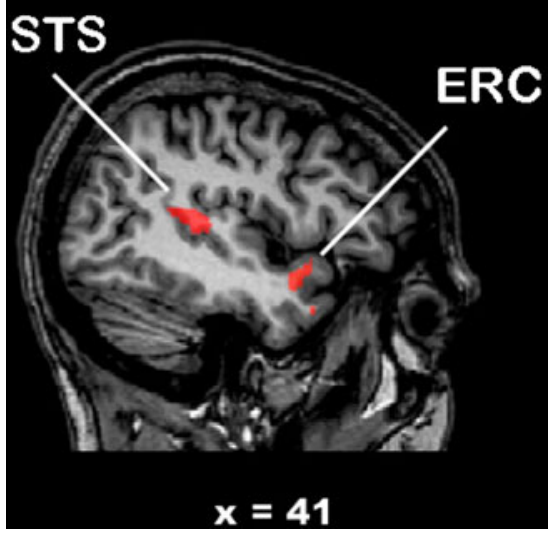

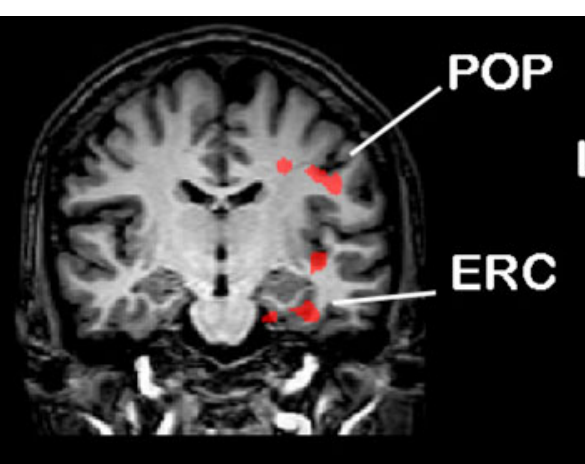

$y=-14$

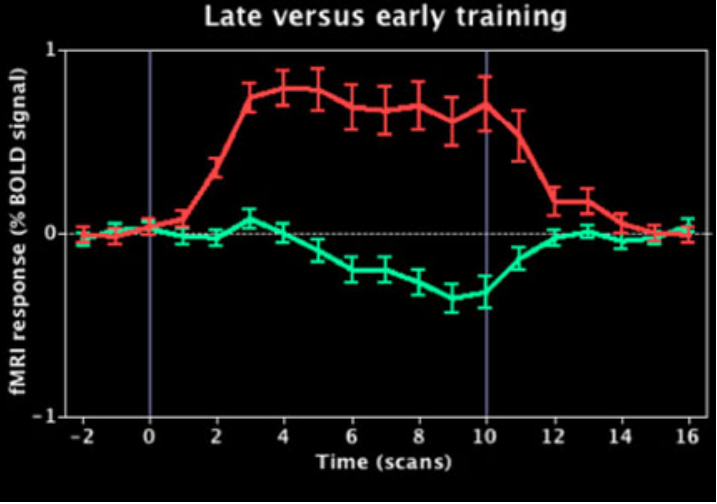


visualizing the previously seen affective images to autobiographical memories of positive events.

Considering the success of the neurofeedback training and the reported strategies of happy memories and positive emotions, we would have expected some positive moodinducing effect of the procedure, which was not the case. One reason might be that the target area was not localized by the contrast between positive and negative emotions, but by the contrast positive vs. baseline, because of the considerable overlap between activations for stimuli with different valence (Fig. 1) and difficulty of finding "positivespecific" areas. This approach was required because of its higher sensitivity and the need to identify target areas in each individual participant. Ideally, one would envisage conducting a procedure like that of Costa et al. (2010) who used a narrative emotional imagery procedure to determine areas of the brain that responded selectively to positive and negative valence. However, given the requirement of the localizer to robustly determine emotion-processing regions in every participant, we chose to use the same procedure as in our earlier study (Johnston et al., 2010) that has so far proven a stable approach for determining regions that respond to positive and negative valence. Similarly, it may prove useful to utilize a procedure for the localizer task that mirrors the main experimental task, i.e., an affective imagery localizer rather than a more visually based procedure. We cannot rule out that such an "internal" localizer procedure (opposed to our "external" emotional stimulation) may have resulted in more lasting effects on mood. The upregulated areas may thus have represented non-specific aspects of emotion (such as arousal) or contain overlapping populations of valence-specific neurons that cannot be differentiated with classical univariate analysis. An improved localizer procedure that separately manipulates arousal and valence and employs multivariate analysis to differentiate valence specific activation patterns (Costa et al., 2010; De Martino et al., 2008; Haynes \& Rees, 2006; Kamitani \& Tong, 2006; Kriegeskorte et al., 2010; Kuncheva et al., 2010) may yield targets for neurofeedback that allow for a more selective regulation of networks that are involved in positive mood. Based on the preliminary success of deep-brain stimulation of the subgenual cingulate cortex and ventral striatum in major depression (Giacobbe, Mayberg, Lozano, 2009; Mayberg et al., 2005) we would expect at least transient mood improvement from neurofeedback control of more refined target areas or patterns. The issue of ROI selection is central to the neurofeedback technique and, so far, has received little direct attention. Our results do highlight an important point about ROI selection and provide some interesting possibilities. Each fMRI voxel inevitably includes vast numbers of neurons, which likely have different functions and specializations, which may lead to overlapping representations for positive and negative emotions. Neurofeedback with a specific cognitive or imagery strategy might differentially activate sub-clusters of specialized neurons within a voxel. In such cases, different subjective strategies can lead to similar success of brain regulation at the spatial resolution of voxels, let alone multi-voxel clusters, but potentially different psychometric effects.

A further consideration is that mood effects using neurofeedback via positive emotional imagery may not be obtainable in individuals who, unlike patients with depression, are capable of normal mood regulation. Our participants all had POMS total mood disturbance scores within 1 standard deviation of a normative adult sample (Nyenhuis, Yamamoto, Luchetta, Terrien, \& Parmentier, 1999). Mood effects of neurofeedback may be easier to obtain and assess in clinical populations where the full dynamic range of the scales can be used. Furthermore, healthy individuals would normally be familiar with the experience of happy memories and positive imagery. Such experience may be dormant for many patients with depression, and a training procedure that engages them with the positive features of their personal lives may help circumvent some of the mental obstacles and resistance. Related to this issue is the selection of a long-term emotional change, i.e., mood, as our behavioral measure. A recent study by Caria and colleagues (Caria et al., 2010) assessed the effect of neurofeedback training of left insula on the subsequent arousal and valence ratings of affective pictures. The procedure utilized an interleaved block design where periods of neurofeedback were interspersed with the rating of an affective stimulus. The immediate effect of successful self-regulation on left insula was seen in more negative ratings of aversive pictures. It is conceivable that with the current procedure we would have seen an analogous enhancement of positive ratings. Another direction for future studies would be to include objective measures as employed by Rota et al. (2009) to probe for emotioncognition interactions.

One aspect of the study that we should perhaps point out is that our feedback training was short, consisting of approx. 20 minutes of training. Although this short training period was sufficient for the participants to gain control over their brain activity, it may have been too short to result in mood improvements. Future studies might thus employ longer neurofeedback schedules (split over different sessions) as well as alternative ways of assessing emotions (physiological and cognitive, in addition to the present selfreport measures). One other consideration is the noxious environment presented by the scanning environment (e.g. noise) that may have attenuated any positive changes in mood. While this is a distinct possibility, the lack of a subsequent decrease in mood in our control group, who did not reliably activate regions of interest within the emotion network, would 
suggest that this does not fully account for our lack of mood change using positive imagery based neurofeedback.

Although the present study did not demonstrate any mood effect of the successful self-regulation of emotion areas, we thus continue to see clinical potential in its development into an adjunct tool to aid cognitive interventions that focus on self-efficacy (Bandura 1997). The ability of participants to learn the successful strategies within a single session of fMRI is encouraging regarding practical applications.

Acknowledgements Funded by the Welsh Assembly Government through grants from the Wales Institute of Cognitive Neuroscience and a RCUK Academic Fellowship to SB. We are grateful to our former Masters students Rebekah Keegan, Debra Ford, Sarah Dabbs, and Michael Evans for help with data analysis.

\section{References}

Bandura, A. (1997). Self-efficacy: The exercise of control. New York: Worth Publishers.

Caria, A., Sitaram, R., Veit, R., Begliomini, C., \& Birbaumer, N. (2010). Volitional control of anterior insula activity modulates the response to aversive stimuli. A real-time functional magnetic resonance imaging study. Biological Psychiatry, 68, 425-432.

Caria, A., Veit, R., Sitaram, R., Lotze, M., Weiskopf, N., Grodd, W., et al. (2007). Regulation of anterior insular cortex activity using real-time fMRI. Neuroimage, 35, 1238-1246.

Costa, V. D., Lang, P. J., Sabatinelli, D., Versace, F., \& Bradley, M. M. (2010). Emotional imagery: Assessing pleasure and arousal in the brain's reward circuitry. Human Brain Mapping, 31(9), 14461457.

De Martino, F., Valente, G., Staeren, N., Ashburner, J., Goebel, R., \& Formisano, E. (2008). Combining multivariate voxel selection and support vector machines for mapping and classification of fMRI spatial patterns. Neuroimage, 43(1), 44-58.

deCharms, R. (2007). Reading and controlling human brain activation using real-time functional magnetic resonance imaging. Trends in Cognitive Sciences, 11, 473-481.

deCharms, R., Christoff, K., Glover, G., Pauly, J., Whitfield, S., \& Gabrieli, J. (2004). Learned regulation of spatially localized brain activation using real-time fMRI. Neuroimage, 21, 436-443.

deCharms, R. C., Maeda, F., Glover, G. H., Ludlow, D., Pauly, J. M., Soneji, D., et al. (2005). Control over brain activation and pain learned by using real-time functional MRI. Proceedings of the National Academy of Sciences of the United States of America, 102, 18626-18631.

Forman, S. D., Cohen, J. D., Fitzgerald, J. D., Eddy, W. F., Mintun, M. A. \& Noll, D. C. (1996). Improved Assessment of significant activation in functional magnetic resonance imagery (fMRI): Use of a cluster-size threshold. Magnetic Resonance in Medicine, 33, 636-647.

Giacobbe, P., Mayberg, H. S., \& Lozano, A. M. (2009). Treatment resistant depression as a failure of brain homeostatic mechanisms: Implications for deep brain stimulation. Experimental Neurology, 219(1), 44-52.

Haynes, J. D., \& Rees, G. (2006). Decoding mental states from brain activity in humans. Nature Reviews. Neuroscience, 7(7), 523-534.
Johnston, S. J., Boehm, S. G., Healy, D., Goebel, R., \& Linden, D. E. J. (2010). Neurofeedback: A promising tool for the self-regulation of emotion networks. Neuroimage, 49(1), 1066-1072.

Kamitani, Y., \& Tong, F. (2006). Decoding seen and attended motion directions from activity in the human visual cortex. Current Biology, 16(11), 1096-1102.

Kriegeskorte, N., Cusack, R., \& Bandettini, P. (2010). How does an fMRI voxel sample the neuronal activity pattern: Compact-kernel or complex spatiotemporal filter? Neuroimage, 49(3), 1965-1976.

Kuncheva, L., Rodriguez, J. J., Plumpton, C. O., Linden, D. E. J., \& Johnston, S. J. (2010). Random subspace ensembles for fMRI Classification. IEEE transactions in medical imaging. IEEE Transactions on Medical Imaging, 29(2), 531-542.

Lang, P. J., Bradley, M. M., \& Cuthbert, B. N. (1999). International affective picture system (IAPS): Technical manual and affective ratings. Gainesville, FL: University of Florida, Center for Research in Psychophysiology.

Lorr, M., McNair, D. M., \& Droppleman, L. F. (1971). POMS ${ }^{\mathrm{TM}}$ profile of mood states. North Tonawanda, NY: Multi-Health Systems Inc.

Mayberg, H. S., Lozano, A. M., Voon, V., McNeely, H. E., Seminowicz, D., Hamani, C., et al. (2005). Deep brain stimulation for treatmentresistant depression. Neuron, 45(5), 651-660.

Mayer, J. S., Roebroeck, A., Maurer, K., \& Linden, D. E. (2010). Specialization in the default mode: Task-induced brain deactivations dissociate between visual working memory and attention. Human Brain Mapping, 31(1), 126-139.

Nyenhuis, D. L., Yamamoto, C., Luchetta, T., Terrien, A., \& Parmentier, A. (1999). Adult and geriatric normative data and validation of the profile of mood states. Journal of Clinical Psychology, 55(1), 79-86.

Poldrack, R. A. (2006). Can cognitive processes be inferred from neuroimaging data? Trends in Cognitive Sciences, 10, 59-63.

Posse, S., Fitzgerald, D., Gao, K., Habel, U., Rosenberg, D., Moore, G. J., et al. (2003). Real-time fMRI of temporolimbic regions detects amygdala activation during single-trial self-induced sadness. Neuroimage, 18, 760-768.

Rota, G., Sitaram, R., Veit, R., Erb, M., Weiskopf, N., Dogil, G., et al. (2009). Self-regulation of regional cortical activity using realtime fMRI: The right inferior frontal gyrus and linguistic processing. Human Brain Mapping, 30, 1605-1614.

Watson, D., Clark, L. A., \& Tellegen, A. (1988). Development and validation of brief measures of positive and negative affect: The PANAS scale. Journal of Personality and Social Psychology, 54, 1063-1070.

Weiskopf, N., Mathiak, K., Bock, S., Scharnowski, F., Veit, R., Grodd, W., et al. (2004a). Principles of a brain-computer interface (BCI) based on real-time functional magnetic resonance imaging (fMRI). IEEE Transactions on Biomedical Engineering, 51, 966-970.

Weiskopf, N., Scharnowski, F., Veit, R., Goebel, R., Birbaumer, N., \& Mathiak, K. (2004b). Self-regulation of local brain activity using real-time functional magnetic resonance imaging (fMRI). Journal of Physiology - Paris, 98, 357-373.

Weiskopf, N., Veit, R., Erb, M., Mathiak, K., Grodd, W., Goebel, R., et al. (2003). Physiological self-regulation of regional brain activity using real-time functional magnetic resonance imaging (fMRI): Methodology and exemplary data. Neuroimage, 19, $577-586$.

Yoo, S., O'Leary, H., Fairneny, T., Chen, N., Panych, L., Park, H., et al. (2006). Increasing cortical activity in auditory areas through neurofeedback functional magnetic resonance imaging. NeuroReport, 17, 1273-1278. 